\section{Changes in visual function after intraocular pressure reduction using antiglaucoma medications}

TS Prata, MV Piassi and LAS Melo Jr

when the three medications studied were compared.

Eye (2009) 23, 1081-1085; doi:10.1038/eye.2008.226; published online 1 August 2008

Keywords: glaucoma; visual function; intraocular pressure; contrast sensitivity

\section{Introduction}

Primary open-angle glaucoma (POAG) is the most common type of glaucoma. Recent projections estimate that approximately 60.5 million people will have glaucoma worldwide in 2010. POAG will be responsible for $74 \%$ of these cases. ${ }^{1}$ Elevated intraocular pressure (IOP) is a major risk factor for this disease, which is characterized by retinal ganglion cell degeneration, optic nerve head changes, and vision loss. ${ }^{2,3}$

The glaucomatous damage to visual function has been considered irreversible. ${ }^{4}$ However, several studies have reported partial recovery from visual field defects in some patients, which is related to the IOP reduction achieved in each eye. . $^{5-8}$

During the natural course of glaucomatous optic neuropathy (GON), certain types of functional vision loss may occur earlier than those detected by standard automated perimetry (SAP) ${ }^{4,9}$ Alterations in contrast sensitivity (CS) have been observed before any visible nerve fiber layer damage or SAP visual field defects. ${ }^{10,11} \mathrm{CS}$ alterations have also been shown to precede significant visual acuity loss. ${ }^{12}$ Several studies have reported an improvement of CS in glaucoma patients after treatment with topical beta-blockers, ${ }^{13}$ alpha-agonists, ${ }^{2}$ carbonic anhydrase inhibitors, ${ }^{14}$ or surgical reduction of IOP. ${ }^{4}$
Department of

Ophthalmology, Federal University of São Paulo, São Paulo, Brazil

Correspondence: TS Prata, Department of Ophthalmology

- Glaucoma Service, Federal University of São Paulo,

1232/71,

Al. Campinas,

São Paulo-SP

01404-001.

Brazil

Tel: 55113885 6661;

Fax: 551138843725

E-mail: tiagoprata@

oftalmo.epm.br

Received: 4 September 2007

Accepted in revised form: 24 June 2008

Published online: 1 August 2008

Financial disclosure: The authors have no financial or proprietary interest in any product mentioned in this article. 
Most studies concerning changes in visual function in POAG have limited sample sizes and the results are controversial. ${ }^{2,3,15,16}$ This prospective study was conducted to investigate changes in visual function related to IOP reduction after treating POAG patients with antiglaucoma medications.

\section{Material and methods}

A prospective, randomized clinical trial was conducted involving 54 POAG patients (54 eyes) from the Federal University of São Paulo, Brazil. The protocol adhered to the tenets of the Declaration of Helsinki and was approved by the Institution Ethics Committee. Written informed consent was obtained from all subjects. We certify that all applicable institutional and governmental regulations concerning the ethical use of human volunteers were followed during this research.

The POAG patients had IOP greater than $21 \mathrm{mmHg}$, typical GON with or without visual field defects, gonioscopy (Goldmann three-mirror lens) disclosing a $360^{\circ}$ normal-appearing open angle, and no apparent ocular or systemic abnormalities that might account for the increased IOP. The exclusion criteria included best-corrected visual acuity (BCVA) of less than 20/80, any sign of secondary glaucoma, significant media opacities, and a history of using oral or topical steroids.

Typical GON findings included a vertical cup-to-disc ratio greater than 0.5 , cup-to-disc ratio asymmetry equal to or greater than 0.2 between eyes, notch and splinter haemorrhage. Visual-field SAP findings (Humphrey Swedish Interactive Threshold Algorithm - Standard 24-2, Carl Zeiss Meditec, Dublin, CA, USA) were considered suggestive of glaucoma when either defects of three or more points in cluster with a probability of less than $5 \%$ in a non-edge localization at the pattern deviation plot were observed, or a pattern standard deviation index with a probability of less than $5 \%$ was found or a normal outside limit result was obtained in the glaucoma hemifield test.

Not all patients were receiving antiglaucoma treatment when included in the study, and they randomly received timolol maleate $0.5 \%$, brimonidine tartrate $0.2 \%$, or travoprost $0.004 \%$ in one randomly selected eye. Allocation was based on computer-generated random numbers and was concealed by using sequentially numbered opaque sealed envelopes. The examiner (M.V.P.) responsible for the visual function tests was masked from the treatment allocation of patients throughout the study.

All patients underwent BCVA test (Snellen chart), visual quality perception analysis (visual analogue scale (VAS)), ${ }^{17}$ Goldmann applanation tonometry, CS test (Functional Acuity Contrast Test, Stereo Optical Co,
USA) and SAP in a random sequence at the baseline and then 4 weeks after glaucoma treatment. The VAS consisted of a 10-mm horizontal line where the patients were asked to mark the quality of their vision from 0 (worst possible - left extreme) to 10 (best possible - right extreme). Both SAP and the CS tests were performed twice at the baseline evaluation with a 10-min interval. The second measurement obtained from each examination was used in the analysis. Patients with IOP higher than $25 \mathrm{mmHg}$ at baseline were re-examined a week after starting treatment. Patients with IOP higher than $21 \mathrm{mmHg}$, a week after using antiglaucoma medication, were excluded.

It was calculated that a sample size of 52 individuals would achieve $80 \%$ power to detect a mean change of $1.0 \mathrm{~dB}$ in the SAP MD index with an estimated standard deviation of the change of $2.5 \mathrm{~dB}$ and a significance level of 0.05 . The paired $t$-test and Wilcoxon signed rank test were used to compare the measurement changes between the baseline and the 4-week treatment visits. The Spearman rank correlation test was performed to analyse the correlation between changes in visual function exams and IOP. One-way analysis of variance and the Kruskal-Wallis test were used to compare measurements obtained from the three treatment groups.

\section{Results}

A total of 54 POAG patients were randomly assigned for the three different types of medication. The recruitment period started in March 2005 and the last follow-up visit was in December 2005. At the baseline visit, 18 patients were allocated to be treated with timolol, 17 with brimonidine, and 19 with travoprost. A week after starting the treatment, four patients (one using timolol and three using brimonidine) were excluded because IOP was greater than $21 \mathrm{mmHg}$. All other participants completed the study, with 17 patients using timolol, 14 using brimonidine, and 19 using travoprost (Table 1).

Four weeks after starting the glaucoma treatment, IOP $(P<0.001)$, VAS $(P=0.045)$, SAP mean deviation $(P=0.02)$, and CS frequencies $(12[P=0.03]$ and 18 $[P=0.002]$ cycles/degree) showed statistically significant changes (Table 2).

When the three medication groups were compared, there was no significant difference concerning all parameters analysed $(P>0.40)$ (Table 3$)$. No significant correlations between IOP and visual function changes were found $(P>0.30)$ (Table 4$)$.

\section{Discussion}

In this study, changes in visual function were observed in glaucoma patients after the start of glaucoma treatment. 
Table 1 Baseline characteristics of the patients that completed the study according to the treatment

\begin{tabular}{lccc}
\hline Variable & Timolol & Brimonidine & Travoprost \\
\hline Number & 17 & 14 & 19 \\
Age (years), mean (SD) & $56.2(11.5)$ & $60.9(13.2)$ & $65.9(10.9)$ \\
Gender (male/female) & $7 / 10$ & $4 / 10$ & $6 / 13$ \\
Race (black/white) & $10 / 7$ & $6 / 8$ & $11 / 8$ \\
C/D ratio, mean (SD) & $0.7(0.13)$ & $0.73(0.15)$ & $0.75(0.11)$ \\
IOP (mmHg), mean (SD) & $25.3(4.9)$ & $24.6(5.1)$ & $24.5(3.6)$ \\
BCVA (log MAR), mean (SD) & $0.18(0.16)$ & $0.27(0.22)$ & $0.24(0.22)$ \\
VAS, mean (SD) & $6.8(2.4)$ & $6.5(1.9)$ & $7.4(2.2)$ \\
& & & $-7.10(7.13)$ \\
SAP & & & $5.17(3.75)$ \\
Mean deviation (dB), mean (SD) & $-6.84(8.24)$ & $-5.45(4.99)$ & $5.20(3.81)$ \\
Pattern SD (dB), mean (SD) & $5.19(4.11)$ & & $1.59(0.18)$ \\
& & & $1.54(0.44)$ \\
Contrast sensitivity & $1.63(0.17)$ & $1.60(0.23)$ & $1.35(0.48)$ \\
1.5 (cycle/degree), mean (SD) & $1.66(0.27)$ & $1.41(0.49)$ & $0.50)$ \\
3 (cycles/degree), mean (SD) & $1.55(0.29)$ & $0.89(0.60)$ & $0.51)$ \\
6 (cycles/degree), mean (SD) & $0.94(0.57)$ & $0.46(0.51)$ & $0.22(0.34)$ \\
12 (cycles/degree), mean (SD) & $0.46(0.46)$ & & \\
18 (cycles/degree), mean (SD) & & \\
\hline
\end{tabular}

$\mathrm{BCVA}$, best-corrected visual acuity; C/D ratio, cup-to-disc ratio; IOP, intraocular pressure; log MAR, logarithmic of minimum angle of resolution; SAP, standard automated perimetry; SD, standard deviation; VAS, visual analogue scale.

Table 2 Intraocular pressure and visual function at baseline and after treatment including all participants

\begin{tabular}{|c|c|c|c|c|}
\hline Variable & Baseline mean (SD) & After treatment mean (SD) & Difference mean (SD) & P-value \\
\hline IOP (mmHg) & $24.8(4.4)$ & $16.9(4.3)$ & $7.8(3.6)$ & $<0.001^{\mathrm{a}}$ \\
\hline BCVA (log MAR) & $0.23(0.20)$ & $0.20(0.19)$ & $-0.03(0.12)$ & $0.06^{\mathrm{b}}$ \\
\hline VAS & $6.96(2.20)$ & $7.52(2.01)$ & $0.56(1.93)$ & $0.045^{\mathrm{b}}$ \\
\hline \multicolumn{5}{|l|}{ SAP } \\
\hline Mean deviation (dB) & $-6.56(6.93)$ & $-5.72(6.73)$ & $0.84(2.45)$ & $0.02^{\mathrm{a}}$ \\
\hline Pattern SD (dB) & $5.19(3.81)$ & $4.95(4.01)$ & $-0.24(1.96)$ & $0.26^{\mathrm{a}}$ \\
\hline \multicolumn{5}{|l|}{ Contrast sensitivity } \\
\hline 1.5 (cycle/degree) & $1.61(0.19)$ & $1.63(0.20)$ & $0.02(0.16)$ & $0.21^{\mathrm{a}}$ \\
\hline 3 (cycles/degree) & $1.58(0.40)$ & $1.65(0.28)$ & $0.07(0.28)$ & $0.21^{\mathrm{a}}$ \\
\hline 6 (cycles/degree) & $1.42(0.44)$ & $1.42(0.52)$ & $-0.003(0.38)$ & $0.24^{\mathrm{a}}$ \\
\hline 12 (cycles/degree) & $0.87(0.55)$ & $0.97(0.56)$ & $0.10(0.37)$ & $0.03^{\mathrm{a}}$ \\
\hline 18 (cycles/degree) & $0.37(0.44)$ & $0.55(0.48)$ & $0.18(0.42)$ & $0.002^{\mathrm{a}}$ \\
\hline
\end{tabular}

BCVA, best-corrected visual acuity; IOP, intraocular pressure; log MAR, logarithmic of minimum angle of resolution; SAP, standard automated perimetry; $\mathrm{SD}$, standard deviation; VAS, visual analogue scale.

${ }^{a}$ Wilcoxon signed rank test.

bPaired $t$-test.

However, these changes were not correlated with IOP reduction. Previous studies have demonstrated an improvement of CS with different medications. ${ }^{2,13}$ Evans et $a l^{2}$ evaluated 16 POAG patients using brimonidine or timolol and reported an improvement of CS (6 and 12 cycles/degree) with brimonidine. There were no significant changes in the group tested with timolol. On the other hand, in this study, patients using brimonidine showed a lower mean IOP $(22.3 \mathrm{mmHg})$ and a better BCVA at baseline. These facts could be related to the differences found between study results.
Analysing visual function changes after surgical reduction of IOP, Gandolfi et $a l^{4}$ have described a significant improvement in CS at frequencies of 3,6, and 12 cycles/degree. They also observed a moderate to strong association between CS changes and IOP reductions at frequencies of $3\left(R^{2}=0.67\right)$ and 12 $\left(R^{2}=0.43\right)$ cycles/degree. The study had a long followup (36 months), and the best threshold of CS was achieved at 9 months. The 10 patients evaluated had high IOP ( $>30 \mathrm{mmHg}$ ), no visual field defects, and BCVA of $20 / 20$ at baseline. The differences in baseline 
Table 3 Changes (after treatment minus baseline) in intraocular pressure and visual function for each treatment group

\begin{tabular}{|c|c|c|c|c|}
\hline Variable & Timolol mean (SD) & Brimonidine mean (SD) & Travoprost mean (SD) & P-value \\
\hline IOP (mmHg) & $-7.8(3.7)$ & $-7.1(3.8)$ & $-8.3(3.6)$ & $0.69^{\mathrm{a}}$ \\
\hline BCVA (log MAR) & $-0.03(0.07)$ & $-0.04(0.18)$ & $-0.04(0.10)$ & $0.84^{\mathrm{b}}$ \\
\hline VAS & $0.23(1.75)$ & $0.78(1.93)$ & $0.68(2.13)$ & $0.41^{\mathrm{b}}$ \\
\hline \multicolumn{5}{|l|}{$S A P$} \\
\hline Mean deviation (dB) & $1.01(2.53)$ & $0.68(2.70)$ & $0.81(2.32)$ & $0.93^{\mathrm{a}}$ \\
\hline Pattern SD (dB) & $-0.84(2.44)$ & $0.36(1.35)$ & $-0.14(1.80)$ & $0.62^{\mathrm{b}}$ \\
\hline \multicolumn{5}{|l|}{ Contrast sensitivity } \\
\hline 1.5 (cycle/degree) & $0.04(0.13)$ & $0.04(0.19)$ & $-0.01(0.16)$ & $0.50^{\mathrm{b}}$ \\
\hline 3 (cycles/degree) & $0.08(0.19)$ & $0.10(0.30)$ & $0.03(0.34)$ & $0.49^{\mathrm{b}}$ \\
\hline 6 (cycles/degree) & $0.03(0.28)$ & $-0.04(0.38)$ & $-0.01(0.48)$ & $0.88^{\mathrm{b}}$ \\
\hline 12 (cycles/degree) & $0.19(0.47)$ & $0.07(0.34)$ & $0.03(0.28)$ & $0.51^{\mathrm{b}}$ \\
\hline 18 (cycles/degree) & $0.26(0.29)$ & $0.13(0.38)$ & $0.16(0.54)$ & $0.41^{\mathrm{b}}$ \\
\hline
\end{tabular}

BCVA, best-corrected visual acuity; IOP, intraocular pressure; log MAR, logarithmic of minimum angle of resolution; SAP, standard automated perimetry; $\mathrm{SD}$, standard deviation; VAS, visual analogue scale.

${ }^{\mathrm{a} O n e-w a y ~ a n a l y s i s ~ o f ~ v a r i a n c e . ~}$

${ }^{\mathrm{b}}$ Kruskal-Wallis test.

Table 4 Correlation between intraocular pressure reduction and visual function changes including all participants

\begin{tabular}{lcc}
\hline Visual function & $r_{s}^{\text {a }}(95 \%$ CI $)$ & P-value \\
\hline BCVA & -0.01 (-0.29 to 0.27$)$ & 0.96 \\
VAS & -0.07 (-0.21 to 0.35$)$ & 0.61 \\
& & \\
SAP & 0.12 (-0.17 to 0.38$)$ & 0.42 \\
$\quad$ Mean deviation & 0.09 (-0.19 to 0.36$)$ & 0.51 \\
Pattern SD & & \\
Contrast sensitivity & & \\
1.5 (cycle/degree) & $-0.15(-0.41$ to 0.13$)$ & 0.30 \\
3 (cycles/degree) & $-0.07(-0.34$ to 0.21$)$ & 0.63 \\
6 (cycles/degree) & $0.10(-0.18$ to 0.37$)$ & 0.49 \\
12 (cycles/degree) & $-0.11(-0.38$ to 0.17$)$ & 0.45 \\
18 (cycles/degree) & $0.14(-0.14$ to 0.41$)$ & 0.32 \\
\hline
\end{tabular}

BCVA, best-corrected visual acuity; CI, confidence interval; SAP, standard automated perimetry; SD, standard deviation; VAS, visual analogue scale. ${ }^{\text {aS }}$ pearman rank correlation test.

measurements, as well as in the follow-up time between the former study and the present one might be responsible for the different outcomes observed for each study.

In our study, POAG patients receiving travoprost treatment did not present any difference in visual function changes when compared with patients receiving the other two medications. Studies with a similar design using prostaglandin analogues were not found in literature. Considering other visual function parameters, no significant changes were observed in the BCVA or in IOP in this study (mean of $24.8 \mathrm{mmHg}$ ) at baseline. However, this fact does not preclude the possibility of improvement in patients with higher IOP levels. A significant difference in the SAP mean deviation index was found, but not in the pattern standard deviation. Although different studies describe an improvement of visual field after reducing IOP in glaucoma eyes, ${ }^{6-8,18}$ this improvement has not been reported by other studies. ${ }^{15,16}$ Heijl et $a l^{15}$ analysed 42 glaucoma eyes after laser trabeculoplasty and found no reduction of visual field defects. In relation to the VAS outcomes, most patients in this study showed better results after glaucoma treatment. This could be due to either a true improvement in the visual function or psychological influence during treatment.

Visual function changes in glaucoma patients have been the main subject of several studies, and the results in literature remain controversial. . $^{2,6,7,13,15,16,18}$ Some possible reasons for the discrepancies found among the studies could be related to sample size, baseline IOP level, disease stage, treatment modality, as well as spontaneous fluctuations of the visual field (long-term fluctuation) and the influence of learning.

The exact mechanism responsible for an improvement in visual function after glaucoma treatment remains unknown. Two main possibilities are improvement of ganglion cell activity and an increase in ocular perfusion. ${ }^{2,4,13}$ During the disease process, subpopulations of cells may be dead, damaged, or healthy. ${ }^{2}$ The improvement in visual function reported here could be due to a recovery of suffering ganglion cells after a decrease in IOP. ${ }^{2,4,13}$ In relation to ocular haemodynamics, previous reports observed better ocular perfusion with the use of hypotensive medications. ${ }^{19,20}$ This improvement in microcirculatory conditions was associated with contrast sensitivity enhancement in different studies, especially at the frequency of 6 cycles/degree. ${ }^{14,20}$ 
Some aspects of this study should be noticed: (1) there is no control group without treatment, due to ethical reasons; (2) most excluded patients, due to lack of IOP control, were on brimonidine, which could bias the results of this medication; (3) two visual fields at baseline may not be enough to exclude the learning effect observed in some patients; (4) the comparison between the three medications should be interpreted cautiously due to the limited sample size of each group; (5) in the light of multiple statistical tests performed, the $P$-values should also be cautiously interpreted due to the possibility of type I error inflation.

In conclusion, this study suggests that patients' visual quality perception, SAP mean deviation index, and CS at higher frequencies improve after starting glaucoma therapy. However, no correlation was found between IOP reduction and changes in visual function, and no differences were found in visual function changes between the three medications studied.

\section{References}

1 Quigley HA, Broman AT. The number of people with glaucoma worldwide in 2010 and 2020. Br J Ophthalmol 2006; 90: 262-267.

2 Evans DW, Hosking SL, Gherghel D, Bartlett JD. Contrast sensitivity improves after brimonidine therapy in primary open angle glaucoma: a case for neuroprotection. $\mathrm{Br} \mathrm{J}$ Ophthalmol 2003; 87: 1463-1465.

3 Weinreb RN, Khaw PT. Primary open-angle glaucoma. Lancet 2004; 363: 1711-1720.

4 Gandolfi SA, Cimino L, Sangermani C, Ungaro N, Mora P, Tardini MG. Improvement of spatial contrast sensitivity threshold after surgical reduction of intraocular pressure in unilateral high-tension glaucoma. Invest Ophthalmol Vis Sci 2005; 46: 197-201.

5 Flammer J, Drance SM. Effect of acetazolamide on the differential threshold. Arch Ophthalmol 1983; 101: 1378-1380.

6 Tsai CS, Shin DH, Wan JY, Zeiter JH. Visual field global indices in patients with reversal of glaucomatous cupping after intraocular pressure reduction. Ophthalmology 1991; 98: 1412-1419.

7 Katz LJ, Spaeth GL, Cantor LB, Poryzees EM, Steinmann WC. Reversible optic disk cupping and visual field improvement in adults with glaucoma. Am J Ophthalmol 1989; 107: 485-492.

8 Gandolfi SA. Improvement of visual field indices after surgical reduction of intraocular pressure. Ophthalmic Surg 1995; 26: 121-126.

9 Stewart WC, Chauhan BC. Newer visual function tests in the evaluation of glaucoma. Surv Ophthalmol 1995; 40 : 119-135.

10 Regan D, Neima D. Low-contrast letter charts in early diabetic retinopathy, ocular hypertension, glaucoma, and Parkinson's disease. Br J Ophthalmol 1984; 68: 885-889.

11 Ross JE, Bron AJ, Clarke DD. Contrast sensitivity and visual disability in chronic simple glaucoma. Br J Ophthalmol 1984; 68: 821-827.

12 Hawkins AS, Szlyk JP, Ardickas Z, Alexander KR, Wilensky JT. Comparison of contrast sensitivity, visual acuity, and Humphrey visual field testing in patients with glaucoma. J Glaucoma 2003; 12: 134-138.

13 Pomerance GN, Evans DW. Test-retest reliability of the CSV1000 contrast test and its relationship to glaucoma therapy. Invest Ophthalmol Vis Sci 1994; 35: 3357-3361.

14 Arend O, Harris A, Wolter P, Remky A. Evaluation of retinal haemodynamics and retinal function after application of dorzolamide, timolol and latanoprost in newly diagnosed open-angle glaucoma patients. Acta Ophthalmol Scand 2003; 81: 474-479.

15 Heijl A, Bengtsson B. The short-term effect of laser trabeculoplasty on the glaucomatous visual field. A prospective study using computerized perimetry. Acta Ophthalmol 1984; 62: 705-714.

16 Holmin C, Krakau CE. Trabeculoplasty and visual field decay: a follow-up study using computerized perimetry. Curr Eye Res 1984; 3: 1101-1105.

17 Pointer JS. Evaluating the visual experience: visual acuity and the visual analogue scale. Ophthalmic Physiol Opt 2003; 23: 547-552.

18 Salim S, Paranhos A, Lima M, Shields MB. Influence of surgical reduction of intraocular pressure on regions of the visual field with different levels of sensitivity. Am J Ophthalmol 2001; 132: 496-500.

19 Evans DW, Harris A, Chung HS, Cantor LB, Garzozi HJ. Effects of long-term hypotensive therapy with nonselective beta-blockers on ocular hemodynamics in primary open-angle glaucoma. J Glaucoma 1999; 8: 12-17.

20 Harris A, Evans DW, Cantor LB, Martin B. Hemodynamic and visual function effects of oral nifedipine in patients with normal-tension glaucoma. Am J Ophthalmol 1997; 124: 296-302. 